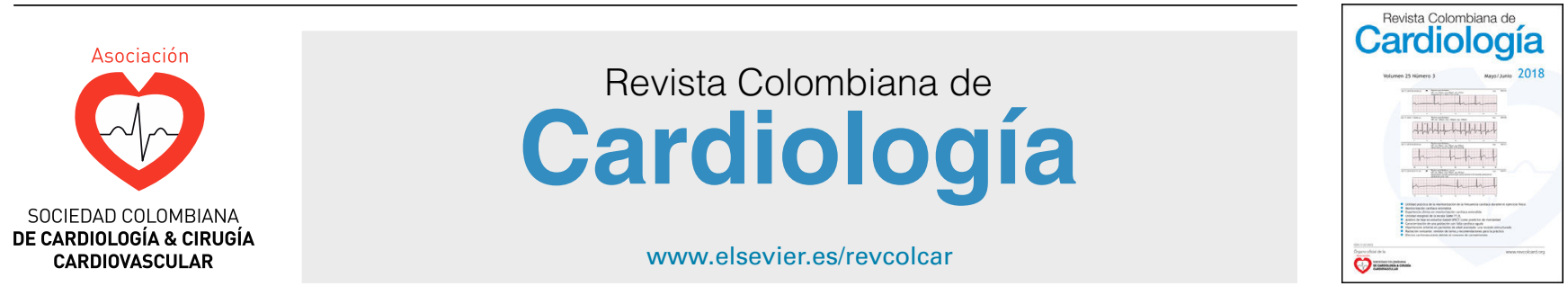

CARDIOLOGÍA DEL ADULTO - ARTÍCULO ORIGINAL

\title{
Bradicardia inducida por quimioterapia en pacientes oncológicos
}

\section{Enrique Ruiz-Mori ${ }^{a, *}$, Leonor Ayala-Bustamante ${ }^{a}$, Jorge Burgos-Bustamante ${ }^{a}$ y Cristian Pacheco Román ${ }^{\text {b }}$}

\author{
a Servicio de Cardiología, Instituto Nacional de Enfermedades Neoplásicas, Lima, Perú \\ b Departamento de Oncología, Instituto Nacional de Enfermedades Neoplásicas, Lima, Perú
}

Recibido el 5 de enero de 2018; aceptado el 4 de agosto de 2018

Disponible en Internet el 29 de octubre de 2018

\section{PALABRAS CLAVE \\ Bradicardia; \\ Arritmias; \\ Quimioterapia; \\ Efecto adverso \\ cardiovascular; \\ Cardio-oncología}

\begin{abstract}
Resumen
Introducción: cada día se reportan efectos tóxicos de la quimioterapia en el corazón, entre ellos las arritmias; sin embargo, las publicaciones sobre bradicardia ocasionada por antineoplásicos son escasas.

Objetivo: describir y analizar la presencia de bradicardia posquimioterapia en el paciente oncológico.

Materiales y métodos: estudio no experimental, descriptivo, retrospectivo, en el que se incluyeron pacientes atendidos durante el año 2017 en un Servicio de Cardiología, a causa de bradicardia posquimioterapia.

Resultados: se evaluaron 59 pacientes, 31 varones $(52,5 \%)$ y 28 mujeres $(47,5 \%)$, con una mediana de edad de 42 años. La mediana de la frecuencia cardiaca fue 46 latidos por minuto. La bradicardia fue más frecuente en leucemia mielocítica aguda $(25,42 \%)$, seguida por leucemia linfoblástica aguda (20,34\%). Fue asintomática en el $88,13 \%$ de los casos. Los fármacos quimioterápicos relacionados con bradicardia en leucemia mielocítica aguda fueron la citarabina en combinación con la daunorubicina, mientras que en leucemia linfoblástica aguda fueron la vincristina en combinación con la daunorubicina. Se presentó intervalo QTc largo en 12 casos (20,34\%). El tiempo entre quimioterapia y el inicio de la bradicardia fue 24 a 48 horas en 35,6\% y la recuperación de la frecuencia cardiaca fue entre 24 a 48 horas en el 61,02\%.

Conclusiones: la bradicardia sinusal como efecto adverso de la quimioterapia, es más frecuente en la leucemia mielocítica aguda, mientras que los medicamentos antineoplásicos relacionados con la bradicardia más comunes fueron la citarabina y la daunorubicina.

(C) 2018 Sociedad Colombiana de Cardiología y Cirugía Cardiovascular. Publicado por Elsevier España, S.L.U. Este es un artículo Open Access bajo la licencia CC BY-NC-ND (http:// creativecommons.org/licenses/by-nc-nd/4.0/).
\end{abstract}

\footnotetext{
* Autor para correspondencia.

Correo electrónico: cruizm@usmp.pe (E. Ruiz-Mori).
} 


\section{KEYWORDS}

Bradycardia;

Arrhythmias;

Chemotherapy;

Cardiovascular

adverse effect;

Cardio-oncology

\section{Chemotherapy-induced bradycardia in oncology patients}

\begin{abstract}
Introduction: There are daily reports of the toxic effects of chemotherapy on the heart, among them are the arrhythmias. However, there are very few publications on bradycardia caused by anti-neoplastic treatment.

Objective: To describe and analyse the presence of post-chemotherapy bradycardia in the oncology patient.

Materials and methods: A non-experimental, descriptive and retrospective study was conducted on patients seen during the year 2017 in a Cardiology Department due to post-chemotherapy bradycardia.

Results: A total of 59 patients were evaluated, of whom 31 (52.5\%) were males and 28 (47.5\%) women, and with a median age of 42 years. The median heart rate was 46 beats per minute. The bradycardia was more common in acute myelocytic leukaemia (25.42\%), followed by acute lymphoblastic leukaemia (20.34\%). It was asymptomatic in $88.31 \%$ of cases. The chemotherapy drugs associated with bradycardia in acute myelocytic leukaemia were cytarabine in combination with daunorubicin, whilst in acute lymphoblastic leukaemia they were vincristine in combination with daunorubicin. A prolonged QTc interval was present in $12(20.34 \%)$ of cases. The time between the chemotherapy and the onset of bradycardia was 24 to 48 hours in $35.6 \%$, and the recovery of the heart rate was between 24 and 48 hours in $61.02 \%$.

Conclusions: Sinus bradycardia as an adverse effect of chemotherapy is more frequent in acute myelocytic leukaemia, whilst the most common anti-neoplastic drugs associated with bradycardia were cytarabine and daunorubicin.

(c) 2018 Sociedad Colombiana de Cardiología y Cirugía Cardiovascular. Published by Elsevier España, S.L.U. This is an open access article under the CC BY-NC-ND license (http:// creativecommons.org/licenses/by-nc-nd/4.0/).
\end{abstract}

\section{Introducción}

En los últimos años cada vez se registran con más frecuencia los efectos tóxicos de la quimioterapia sobre el corazón, ocasionando en muchos casos alta mortalidad ${ }^{1}$. Al conocido daño de la función cardiaca por las antraciclinas se han incorporado fenómenos de vasoespasmo coronario, hipertensión arterial, procesos trombóticos/tromboembólicos y arritmias $^{2}$.

La incidencia y la prevalencia de las arritmias cardiacas en la población oncológica secundaria a quimioterapia no ha podido determinarse con exactitud, en cuyo caso se considera que múltiples circunstancias propias de la enfermedad y sus complicaciones, o del paciente y su medio, o del fármaco y terapias adyuvantes, condicionan que no exista una causalidad clara ${ }^{3}$.

Los fármacos oncológicos que con mayor frecuencia se relacionan con arritmias cardiacas son: antraciclinas, ifosfamida, cisplatino, talidomida, 5-fluoruracilo, paclitaxel, y recientemente trastuzumab, entre otros; ocasionan diversos trastornos del ritmo, desde las bradicardias asintomáticas hasta las taquiarritmias sintomáticas, incluyendo a la fibrilación auricular ${ }^{4,5}$.

Las taquiarritmias, al ser generalmente sintomáticas, son las que más se detectan en los pacientes oncológicos; así, se reporta que las antraciclinas pueden ocasionar fibrilación auricular paroxística en 1,3 a 10,3\% en las primeras 24 horas de infusión; altas dosis de ciclofosfamida e ifosfamida se asocian con taquicardias supraventriculares, o que el uso de melfalan previo al trasplante de médula produce fibrilación auricular en un 6,6 a $8,3 \%$. Sin embargo, se reporta poca información sobre bradiarritmias ${ }^{6,7}$.

El objetivo general de este estudio es describir y analizar la frecuencia de las bradicardias que aparecen luego de la administración de agentes quimioterápicos en el paciente oncológico del Instituto Nacional de Enfermedades Neoplásicas (INEN).

\section{Materiales y métodos}

Se llevó a cabo un estudio de tipo no experimental, descriptivo, retrospectivo, en el que se incluyeron pacientes atendidos en los consultorios del Servicio de Cardiología, que presentaron un episodio de bradicardia luego de la administración de quimioterapia, durante el periodo del 2 de enero al 31 de diciembre de 2017. Se consideró bradicardia a toda condición en la que la frecuencia cardiaca fuera menor o igual de 50 latidos por minuto.

Se excluyeron aquellos que previo a la quimioterapia cursaran con bradicardia; o que recibieran alguna medicación que les pudiera ocasionar bradicardia, o tuvieran diagnóstico previo de bradiarritmia.

El cálculo del intervalo QT corregido (QTc) se hizo según la fórmula de Bazett y se consideró intervalo QT largo si era mayor a 440 milisegundos (ms) en hombres y mayor a $450 \mathrm{~ms}$ en mujeres ${ }^{8}$. 


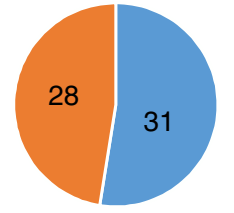

$$
\text { Varones Mujeres }
$$

Figura 1 Número de pacientes por género.

\section{Resultados}

Se estudiaron 59 pacientes que fueron derivados del servicio de Medicina Oncológica al servicio de Cardiología por bradicardia luego de la administración de quimioterapia; 31 de estos eran varones $(52,5 \%)$ y 28 mujeres $(47,5 \%)$ (fig. 1$)$, con una mediana de edad de 42 años (oscilación de 5 a 77 años) (fig. 2).

La mediana de la frecuencia cardiaca registrada en estos pacientes luego de la quimioterapia fue de 46 latidos por minuto. La frecuencia cardiaca más baja fue de 35 latidos por minuto y se registró en un paciente varón con cáncer de páncreas que recibió capecitabina, oxaliplatino y gemcitabina, en quien hubo un descenso de la frecuencia de un 48,5\% (porcentaje de reducción de la frecuencia basal de 68 latidos por minuto) (tabla 1).

El porcentaje de reducción de la frecuencia cardiaca con relación al valor basal (frecuencia cardiaca basal versus frecuencia cardiaca evento) fluctuó desde un $18,33 \%$ hasta un $56,86 \%$, con una mediana de $38,46 \%$.

La bradicardia sinusal fue más frecuente en aquellos pacientes con diagnóstico de leucemia mielocítica aguda (25,42\%), seguida por leucemia linfoblástica aguda $(20,34 \%)$ y finalmente por cáncer de mama (15,25\%).
La bradicardia se diagnosticó inicialmente al registrar las funciones vitales, sin referir sintomatología alguna, en un $88,13 \%$ de los casos. Sólo 7 pacientes $(11,87 \%)$ refirieron sintomatología, siendo la astenia el síntoma más frecuente, seguida por mareos y visión borrosa. El paciente que alcanzó la frecuencia cardiaca más baja presentó sintomatología e hipotensión arterial que requirió tratamiento médico respectivo. En ninguno de los casos evaluados fue necesario el uso de marcapasos (tabla 2).

Los fármacos quimioterápicos que estuvieron administrándose a los pacientes con leucemia mielocítica aguda antes de presentar bradicardia sinusal fueron la citarabina (ARA-C) (14 de 15 pacientes), en conjunto con daunorubicina (10 de 15), mientras que en leucemia linfoblástica aguda fue la vincristina (8 de 11), asociada con mayor frecuencia con un antraciclínico (daunorubicina) (5 de 11$)$. Siete pacientes recibían un taxano como quimioterapia. Finalmente, 6 pacientes con mieloma múltiple que desarrollaron bradicardia sinusal, recibieron talidomida.

Se detectó intervalo QTc largo en 12 casos $(20,34 \%)$ al desarrollar bradicardia sinusal, el cual revirtió al normalizar la frecuencia cardiaca. No existió diferencia por género, tipo de cáncer o quimioterápico con relación a la prolongación del QT. La mayor duración del QTc fue de $486 \mathrm{~ms}$ en un paciente que alcanzó una frecuencia cardiaca de 44 latidos por minuto, padecía cáncer de ano y recibió 5-fluorouracilo como parte del tratamiento (tabla 1).

En relación con el tiempo transcurrido entre la quimioterapia y el inicio de la bradicardia fue entre 24 a 48 horas la mayor frecuencia $(35,6 \%)$, seguido por aquellos cuya presentación fue entre 3 a 7 días (23,73\%) (tabla 3).

El tiempo de recuperación de la bradicardia a ritmo superior de 60 latidos por minuto, fue con mayor frecuencia entre 24 a 48 horas en un 61,02\%; en 2 casos la frecuencia cardiaca permaneció menor a 60 latidos por minuto (tabla 3).

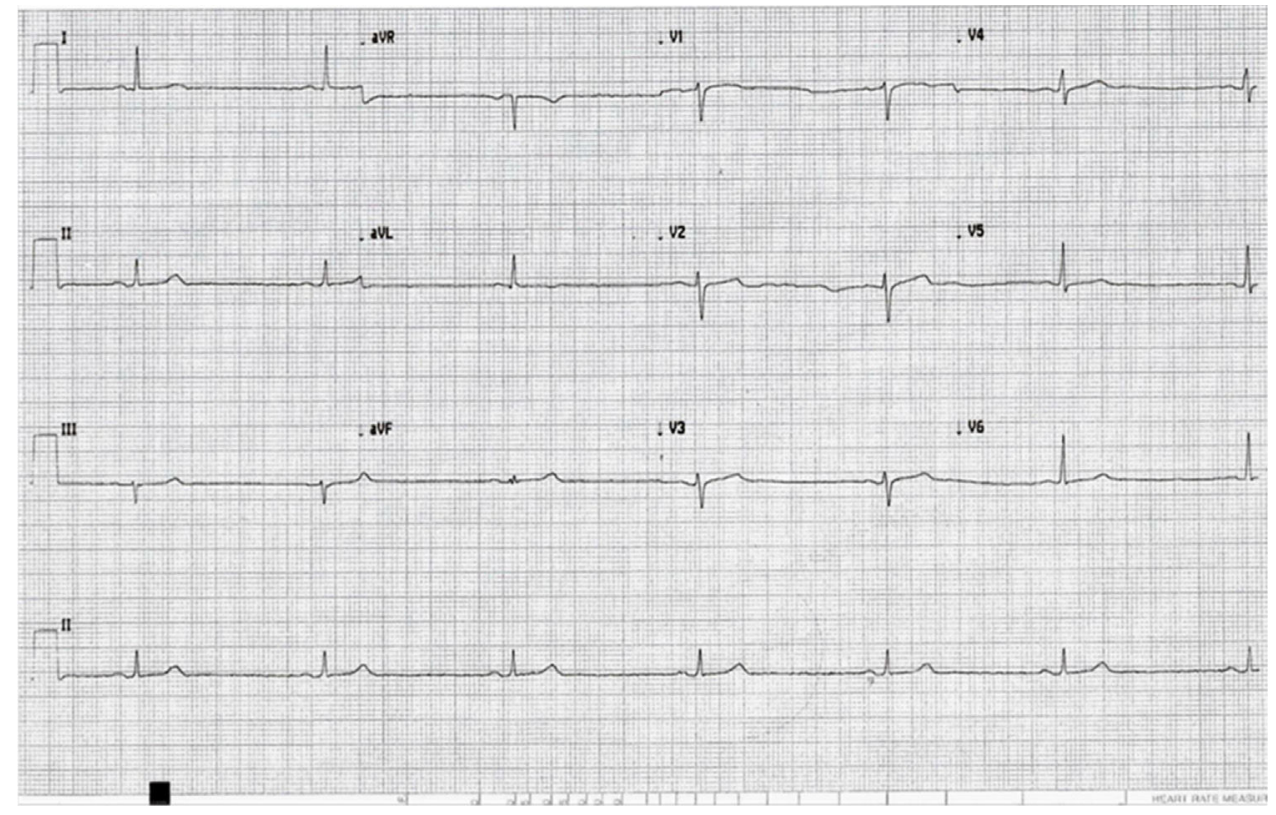

Figura 2 Paciente mujer 56 años. Cáncer de mama. Tratamiento: trastuzumab. Frecuencia cardiaca 38 lat/min PR165 ms QRS $82 \mathrm{~ms}$ QT $429 \mathrm{~ms}$ QTc $351 \mathrm{~ms}$. 
Tabla 1 Características de los pacientes que presentaron bradicardia

\begin{tabular}{|c|c|c|c|c|c|c|c|c|c|}
\hline$\overline{\mathrm{ED}}$ & $\mathrm{S}$ & Diagnóstico & Tratamiento & $\begin{array}{l}\mathrm{FC} \\
\text { basal }\end{array}$ & $\begin{array}{l}\mathrm{FC} \\
\text { evento }\end{array}$ & $\begin{array}{l}\text { Recup. } \\
\text { FC }\end{array}$ & $\begin{array}{l}\text { \% Reduc } \\
\text { FC }\end{array}$ & $\begin{array}{l}\text { Basal } \\
\mathrm{QT} / \mathrm{QTC}\end{array}$ & $\begin{array}{l}\text { Evento } \\
\text { QT/QTC }\end{array}$ \\
\hline 5 & $\mathrm{~F}$ & LLA & VCR/Dauno & 70 & 47 & 79 & 32,85 & $410 / 437$ & $420 / 425$ \\
\hline 44 & $M$ & MM & Talidomida & 62 & 44 & 84 & 29,03 & $401 / 407$ & $483 / 434$ \\
\hline 71 & $\mathrm{~F}$ & LNH: Gástrico & Ciclof/Doxo/VCR & 77 & 44 & 79 & 42,85 & $374 / 425$ & $430 / 429$ \\
\hline 56 & $\mathrm{~F}$ & Ca Mama & Trastuzumab & 65 & 38 & 60 & 41,53 & $439 / 394$ & $429 / 351$ \\
\hline 10 & $M$ & LMA & Citar/Dauno/Etop & 93 & 38 & 88 & 59,13 & $369 / 420$ & $492 / 415$ \\
\hline 10 & $M$ & LMA & Citar/Mitoxant & 72 & 43 & 87 & 40,27 & $390 / 415$ & $475 / 422$ \\
\hline 13 & $\mathrm{~F}$ & LLA & VRC/L-Aspa & 84 & 48 & 79 & 42,85 & $393 / 432$ & $345 / 386$ \\
\hline 29 & $M$ & LMA & Citar/Dauno & 67 & 44 & 71 & 34,32 & $384 / 402$ & $524 / 476$ \\
\hline 72 & $M$ & NM Páncreas & Gemcit/Oxal/Capecit & 68 & 35 & 66 & 48,52 & $391 / 408$ & $432 / 459$ \\
\hline 11 & $M$ & LLA & VCR/Dauno & 70 & 50 & 86 & 28,50 & $394 / 416$ & $471 / 444$ \\
\hline 37 & $\mathrm{~F}$ & LM Crónica & Citar/Dauno/Imat & 62 & 43 & 78 & 30,64 & $395 / 405$ & $511 / 457$ \\
\hline 62 & $M$ & MM & Talidomida & 76 & 46 & 82 & 39,47 & $364 / 395$ & $404 / 370$ \\
\hline 50 & $M$ & LMA & Citar/Dauno & 73 & 43 & 73 & 41,09 & $375 / 401$ & $442 / 387$ \\
\hline 31 & $\mathrm{~F}$ & LMA & Citar/Mitoxant & 64 & 50 & 65 & 21,87 & $387 / 397$ & $452 / 437$ \\
\hline 12 & $\mathrm{~F}$ & LMA & L-Asp/VCR/Dauno & 102 & 44 & 73 & 56,86 & $384 / 435$ & $429 / 409$ \\
\hline 74 & $M$ & Ca de Ano & Capecit/Mitomicina & 72 & 44 & 80 & 38,88 & $415 / 438$ & $452 / 486$ \\
\hline 32 & $\mathrm{~F}$ & Ca Mama & Doxo/Ciclof/Paclit & 89 & 50 & 85 & 43,82 & $405 / 452$ & $429 / 403$ \\
\hline 62 & $\mathrm{~F}$ & Ca Mama & Trastuzumab & 83 & 42 & 72 & 49,39 & $362 / 402$ & $463 / 396$ \\
\hline 42 & $M$ & LNH & Citar/Ritu/Cispl & 72 & 48 & 92 & 33,33 & $380 / 400$ & $437 / 416$ \\
\hline 46 & $M$ & LMA & Citar/Dauno & 78 & 48 & 74 & 38,46 & $399 / 432$ & $435 / 401$ \\
\hline 24 & $\mathrm{~F}$ & LNH & Cispl/Citar/Dexa & 61 & 45 & 74 & 26,22 & $438 / 441$ & $503 / 459$ \\
\hline 18 & $M$ & LLA & Citar/Ciclof/VCR/L-Asp & 70 & 46 & 78 & 34,28 & $314 / 337$ & $428 / 386$ \\
\hline 62 & $M$ & Ca Íleon & Capecit/Oxal & 70 & 49 & 84 & 30,0 & $357 / 377$ & $437 / 408$ \\
\hline 56 & $\mathrm{~F}$ & Ca Mama & Trastuzumab & 73 & 39 & 85 & 46,57 & $360 / 385$ & $473 / 401$ \\
\hline 68 & $M$ & $M M$ & Talidomida/Ciclof/Dexa & 64 & 42 & 89 & 34,37 & $434 / 444$ & $458 / 401$ \\
\hline 53 & $M$ & NM Gástrico & 5-FU/Oxal/Docet & 67 & 48 & 60 & 28,35 & $417 / 432$ & $456 / 421$ \\
\hline 43 & $M$ & LMA & Citar/Dauno & 68 & 47 & 78 & 30,88 & $367 / 389$ & $511 / 472$ \\
\hline 62 & $\mathrm{~F}$ & MM & Talidomida & 63 & 46 & 62 & 26,98 & $425 / 432$ & $445 / 406$ \\
\hline 33 & $\mathrm{~F}$ & Ca mama & Trastuzumab & 85 & 47 & 78 & 44,70 & $358 / 400$ & $460 / 422$ \\
\hline 69 & $M$ & MM & Talidomida/Bortez & 78 & 48 & 78 & 38,46 & $377 / 412$ & $438 / 403$ \\
\hline 34 & $M$ & Linfoma T Ang & Ciclof/MESNA/G CSF & 78 & 49 & 66 & 37,17 & $399 / 432$ & $443 / 438$ \\
\hline 63 & $M$ & Ca Orofaringe & 5-FU/Cispl/Docet & 87 & 47 & 92 & 45,97 & $340 / 415$ & $461 / 422$ \\
\hline 24 & $M$ & TCG & Paclit/Ifos/MESNA & 76 & 47 & 61 & 38,15 & $422 / 452$ & $434 / 396$ \\
\hline 72 & $M$ & LNH Cervical & Ritux/Ciclof/Adria/VCR & 72 & 48 & 76 & 33,33 & $376 / 413$ & $441 / 408$ \\
\hline 54 & $M$ & Ca Pene & Ifos/MESNA/Plat/Paclit & 69 & 41 & 81 & 40,57 & $373 / 392$ & $462 / 400$ \\
\hline 44 & $M$ & LMA & Citar/Dauno & 77 & 46 & 74 & 40,25 & $367 / 399$ & $471 / 427$ \\
\hline 70 & $\mathrm{~F}$ & LNH & VCR/Ciclof/Doxo & 64 & 44 & 62 & 31,25 & $418 / 427$ & $403 / 387$ \\
\hline 5 & $\mathrm{~F}$ & LLA & VCR/Ciclof/Citar & 68 & 47 & 64 & 30,88 & $369 / 386$ & $487 / 450$ \\
\hline 52 & $\mathrm{~F}$ & LLA & L-Asp/VCR/Dauno & 66 & 44 & 67 & 33,33 & $411 / 425$ & $463 / 413$ \\
\hline 26 & $\mathrm{~F}$ & Ca Mama & Paclit & 60 & 49 & 70 & 18,33 & $435 / 436$ & $437 / 406$ \\
\hline 25 & $\mathrm{~F}$ & $\mathrm{LH}$ & Gemcit/Oxal & 61 & 45 & 74 & 26,22 & $438 / 441$ & $503 / 459$ \\
\hline 46 & $M$ & LMA & Citar/Dauno & 64 & 48 & 80 & 25,0 & $440 / 427$ & $435 / 401$ \\
\hline 10 & $M$ & LMA & Citar/Mitoxant & 79 & 38 & 71 & 51,89 & $408 / 443$ & $492 / 415$ \\
\hline 75 & $M$ & Melanoma & Imatinib & 81 & 44 & 75 & 45,67 & $346 / 383$ & $488 / 439$ \\
\hline 13 & $\mathrm{~F}$ & LLA & L-Asp/VCR/Dauno & 82 & 47 & 84 & 42,68 & $358 / 397$ & $493 / 455$ \\
\hline 50 & $M$ & LMA & Citar/Dauno & 87 & 43 & 65 & 50,57 & $360 / 404$ & $358 / 351$ \\
\hline 34 & $\mathrm{~F}$ & Leuc Prom Ag & Dauno/Ac Transret & 99 & 50 & 79 & 49,49 & $392 / 448$ & $443 / 419$ \\
\hline 77 & $\mathrm{~F}$ & Ca Mama & Ciclof/Adria & 64 & 50 & 68 & 21,87 & $399 / 408$ & $440 / 425$ \\
\hline 22 & $M$ & Linfoma Linfo & Citar/MTX/Mitoxant & 77 & 40 & $643 / 4$ & 48,05 & $374 / 406$ & $463 / 396$ \\
\hline 9 & $\mathrm{~F}$ & LMA & Citar/Tioguanina & 63 & 50 & 60 & 20,63 & $430 / 436$ & $419 / 385$ \\
\hline 56 & $M$ & $M M$ & Ciclof/Dexa/Talidomida & 75 & 50 & 58 & 33,33 & $375 / 404$ & $408 / 397$ \\
\hline 42 & $\mathrm{~F}$ & Ca Mama & Paclit & 81 & 46 & 66 & 43,20 & $369 / 406$ & $502 / 461$ \\
\hline 59 & $\mathrm{~F}$ & LMA & Citar/Dauno & 78 & 45 & 66 & 42,30 & $383 / 416$ & $444 / 400$ \\
\hline 39 & $\mathrm{~F}$ & LLA & Citar/MTX/Mercapt & 68 & 48 & 70 & 29,41 & $352 / 369$ & $465 / 431$ \\
\hline 56 & $\mathrm{~F}$ & LLA & Citar/MTX/Mercapt & 62 & 43 & 63 & 30,64 & $377 / 382$ & $465 / 413$ \\
\hline 12 & $M$ & LLA & L-Asp/VCR/Dauno & 72 & 50 & 88 & 30,55 & $411 / 435$ & $471 / 444$ \\
\hline 25 & $\mathrm{~F}$ & Ca Mama & Trastuzumab & 74 & 40 & 80 & 45,94 & $408 / 436$ & $450 / 383$ \\
\hline 30 & $\mathrm{~F}$ & LLA & Imatinib & 92 & 44 & 59 & 52,17 & $340 / 386$ & $481 / 464$ \\
\hline 41 & $M$ & LMA & Citar/Dauno & 80 & 45 & 64 & 43,75 & $380 / 418$ & $405 / 408$ \\
\hline
\end{tabular}

ED: edad. S: sexo. F: femenino. M: masculino. FC: frecuencia cardiaca. RECUP FC: recuperación de la frecuencia cardiaca. \% REDUC FC: porcentaje de reducción de la frecuencia cardiaca. QT: intervalo QT (ms). QTc: intervalo QT corregido (ms).

LLA: leucemia linfoblástica aguda. MM: mieloma múltiple. LNH: linfoma no Hodgkin. LMA: leucemia mielocítica aguda. LM crónica: leucemia mielocítica crónica. Linfoma T Ang: linfoma T angioinmunoblástico. TCG: tumor de células germinales. Leuc Prom Ag: leucemia promielocítica aguda. Linfoma Linfo: linfoma linfoblástico de células T.

Ac. Transret: ácido transretinoico. Adria: adriamicina. Bortez: bortezomib. Capecit: capecitabina. Ciclof: ciclofosfamida. Citar: citarabina. Cispl: cisplatino. Dauno: daunorubicina. Dexa: dexametasona. Doxo: doxorubicina. Etop: etopósido. G CSF: filgastrim. Gemcit: gemcitabina. Ifos: ifosfamida. Imat: imatinib. L-Asp: L-asparaginasa. Mercapt: mercaptopurin. MTX: metotrexate. Mitoxant: mitoxantrona. Oxal: oxaiplatino. Paclit: paclitaxel. Plat: platino. Ritux: rituximab. VCR: vincristina 
Tabla 2 Sintomatología según género

\begin{tabular}{llll}
\hline & Hombres & Mujeres & TOTAL \\
\hline SINTOMATOLOGÍA & 3 & 4 & $7(11,87 \%)$ \\
Astenia & 2 & 3 & \\
Mareos & 2 & 2 & \\
Visión borrosa & 1 & 2 & \\
Hipotensión & 1 & 0 & \\
ASINTOMÁTICO & 28 & 24 & $52(88,13 \%)$ \\
\hline
\end{tabular}

Tabla 3 Tiempo de inicio de la bradicardia y tiempo de recuperación

\begin{tabular}{llll}
\hline $\begin{array}{l}\text { Tiempo de } \\
\text { Inicio de } \\
\text { Bradicardia }\end{array}$ & Casos & $\begin{array}{l}\text { Tiempo de } \\
\text { Recuperación }\end{array}$ & Casos \\
\hline$<24$ horas & $11(18,64 \%)$ & $<24$ horas & $0(0 \%)$ \\
$24-48$ horas & $28(47,46 \%)$ & $24-48$ horas & $36(61,02 \%)$ \\
$48-72$ horas & $4(6,78 \%)$ & $48-72$ horas & $9(15,25 \%)$ \\
3-7 días & $14(23,73 \%)$ & $3-7$ días & $8(13,56 \%)$ \\
& $2(3,39 \%)$ & $>7$ días & $4(6,78 \%)$ \\
$>7$ días & & No se recupera & $2(3,39 \%)$ \\
\hline
\end{tabular}

\section{Discusión}

La cardiotoxicidad irreversible que se observa con el uso de antraciclinas, así como la presencia de alteración de la función ventricular con trastuzumab, son reconocidas como las complicaciones cardiacas más importantes producidas por los fármacos antineoplásicos; no obstante, los trastornos del ritmo cardiaco que ocurren durante la administración de los antineoplásicos o inmediatamente después, cada vez se está haciendo más frecuente, más aún si se considera que el cáncer por sí sólo genera un ambiente propicio para las arritmias 9 .

Las arritmias, también denominadas disritmias, engloban una serie de trastornos del ritmo cardiaco, entre los cuales las taquiarritmias son las más frecuentes, quizá porque generan sintomatología y se detectan más; entre éstas la fibrilación auricular es la que más se reporta en las diversas series, generalmente como consecuencia del uso de agentes alquilantes como el cisplatino o la ciclofosfamida ${ }^{10}$.

En contraste con las taquiarritmias, la bradicardia y las bradiarritmias, o ambas, como complicación de la quimioterapia en pacientes con cáncer está poco documentada, considerándose que con mayor frecuencia es un hallazgo de la evaluación clínica y no porque genere sintomatología, como se ha corroborado en este estudio.

Los diversos disturbios del ritmo cardiaco que se presentan en relación con el tratamiento quimioterápico son por lo general transitorios como ocurre en este reporte, en el que en solo 2 casos persistió la bradicardia.

Determinar el mecanismo por el cual se desarrollan las arritmias es muy complejo, pues la enfermedad por sí misma propicia situaciones arritmogénicas, aunado al agente quimioterápico, el cual según la dosis, la vía de administración o las interacciones, puede determinar la presencia de arritmias. Así mismo, a ello se le suman trastornos electrolíticos o del medio interno que determinarían un medio para que el paciente desarrolle cambios en el ritmo cardiaco, lo cual hace más confuso establecer el proceso fisiopatológico ${ }^{11}$. También se deben considerar los procesos de fibrosis producto de la edad o como consecuencia de la radioterapia, hecho que hace más complejo su análisis etiológico.

La citarabina ha sido un medicamento ampliamente utilizado, que tiene efectos adversos como la mielosupresión (toxicidad hematológica) o la toxicidad gastrointestinal; no obstante, son pocos los casos de bradicardia reportados, como por ejemplo el caso de un paciente con leucemia mielocítica aguda que durante la terapia de inducción presentó bradicardia, o el reporte en un enfermo de linfoma no Hodgkin que precisó descontinuar la infusión de citarabina y requirió el uso de atropina. El probable mecanismo de esta respuesta a la citarabina estaría asociado con un proceso de respuesta inmune o por una reacción de hipersensibilidad ${ }^{12}$.

La vincristina, un agente antimicrotubular (que evita la división celular durante la metafase), pertenece a la clase de fármacos quimioterápicos llamados alcaloides vegetales o alcaloides de la vinca; en este trabajo es el medicamento de mayor uso en leucemia mielocítica aguda combinado con antraciclinas, que han desarrollado episodios de bradicardia sinusal. La vincristina tiene un efecto adverso sobre el sistema nervioso. Las manifestaciones de esta neurotoxicidad corresponden a disfunción motora sensorial, como parestesias y dolores musculares, mientras que dentro de las manifestaciones cardiacas, están las fluctuaciones de la frecuencia cardiaca, esto último como irregularidades del tono parasimpático ${ }^{13,14}$.

Antraciclinas como la daunorubicina, son las drogas antitumorales más utilizadas en el mundo, y su eficacia está claramente demostrada, pero son dosis-dependiente y a la vez esta propiedad es su mayor obstáculo, pues a más dosis, mayor riesgo de desarrollar cardiotoxicidad ${ }^{15}$. Entre los efectos adversos agudos conocidos de las antraciclinas están las arritmias cardiacas, que se pueden presentar entre un 11 a 41\%; entre ellas se destacan la fibrilación auricular, las contracciones prematuras supraventriculares y ventriculares, las taquicardias y, con menor frecuencia, los episodios de bradicardia, estos últimos como consecuencia de bloqueos aurículoventriculares de segundo y tercer grado $^{16}$. Los mecanismos propuestos estarían en relación con la interacción con canales iónicos, los cuales también pueden generar cambios en el intervalo QT, prolongándolo. Recientemente se ha descrito bradicardia en la forma liposomal de doxorrubicina, preparación que tiene por objeto disminuir los efectos cardiotóxicos de las antraciclinas ${ }^{17}$.

Por otro lado, la bradicardia sinusal asintomática que tiene lugar con el uso de taxanos, es un fenómeno relativamente frecuente ( $30 \%$ de casos), mientras que los casos severos que pueden llevar al síncope, son poco comunes (5\%). De otro lado, al tener efecto directo sobre el sistema Purkinje, paclitaxel es considerado como un medicamento prototipo de arritmia cardiaca y por otro lado su efecto indirecto de liberar histamina permite explicar la bradicardia en algunos de los usuarios, en comparación con docetaxel con el que se describen taquicardia sinusal y aleteo auricular, entre otras arritmias ${ }^{2,18}$.

La talidomida se sintetizó en 1953 y se empezó a comercializar en 1957 como un sedante. Fue hasta 1961, que el profesor Lenz publicó la relación posible entre talidomida 
y graves malformaciones observadas en recién nacidos de madres que habían consumido este fármaco, lo que finalmente obligó a descontinuar su uso. Sin embargo, en 1997 Dámato y Kenyon reportaron que tenía propiedades antiangiogénicas al inhibir el factor de crecimiento vascular endotelial y el factor de crecimiento de fibroblastos ${ }^{19}$, permitiendo posteriormente que Bart Barlogie lo posicionara como droga de elección en mieloma múltiple. En el presente trabajo se registró bradicardia sinusal asociada a talidomida, efecto adverso que en la literatura médica se reporta en un $27 \%$, hasta un $53 \%$ de casos, y con el que es frecuente que no se ocasione ninguna sintomatología ${ }^{20}$. Sin embargo se describen casos puntuales de bloqueo de tercer grado o bloqueo aurículo-ventricular completo que puede ocasionar hipotensión arterial y pérdida de conciencia, como sucedió con un paciente que inició talidomida dos semanas antes y que tenía un electrocardiograma normal en la conducción aurículo-ventricular y no recibía otro fármaco. Los efectos de la talidomida sobre el corazón están relacionados con el factor de necrosis tumoral (TNF) y la interleucina 6 (IL-6). La insuficiencia cardiaca reportada por el uso de este fármaco se asocia con fenómenos de apoptosis regulados por el TNF, así como con la generación de estrés oxidativo y la inducción de disfunción endotelial. Mientras que la IL-6 modularía indirectamente la presencia de bradicardia, la administración de IL-6 recombinante en forma experimental puede producir fibrilación auricular en pacientes con neoplasia maligna. En pacientes con mieloma múltiple la talidomida reduce las concentraciones de IL-6, lo cual determinaría la presencia de bradicardia. También se ha planteado que en algunos casos se puede inducir hipotiroidismo condicionando bradicardia o también el depósito de amiloide sobre el corazón, con lo cual se propician episodios de bradicardia ${ }^{21,22}$.

El cisplatino es otro medicamento que está relacionado con bradicardia, desde que en 1983 Schlaeffer reportó en un paciente episodios repetidos de bradicardia sinusal durante el uso de este fármaco, hasta reportes actuales que describen frecuencias cardiacas de 35 latidos por minuto ${ }^{5}$. En 2012 se reportaron sólo 12 casos de bradicardia inducida por cisplatino a la Food and Drug Administration (FDA). Cuando el paciente refiere este efecto adverso lo reporta generalmente durante las primeras dosis ${ }^{23}$. En el presente reporte sólo se observó en 3 pacientes. El mecanismo de la arritmia podría ser una alteración del canal de sodio. ${ }^{24}$

La prolongación del QTc es un marcador de mal pronóstico y de riesgo de muerte súbita. En esta serie existieron 12 casos y no se pudo relacionar con un fármaco específico. Está demostrado que los antineoplásicos prolongan el intervalo QTc, como el trióxido de arsénico, el cual se usa en casos de leucemia y en donde se ha registrado hasta un 30 a $40 \%$ de frecuencia. El mecanismo de este efecto adverso se relaciona con trastornos electrolíticos que se producen por diarreas o vómitos concomitantes. Otro fármaco que ocasiona prolongación de QT es el 5-fluorouracilo. El uso de antraciclinas también afecta el sistema eléctrico del corazón. En fibras de Purkinje canino, las antraciclinas prolongan el potencial de acción; así mismo se ha observado en ratas que se puede producir ensanchamiento del complejo QRS y prolongación del QTc ${ }^{5,10}$. De otra parte, se ha descrito que nuevas drogas como nilotinib, dasatinib o sunitinib pueden prolongar el intervalo $\mathrm{QT}^{25}$.
En todos los casos ha revertido espontáneamente, sin embargo es necesario monitorizar a los pacientes a fin de observar que no progrese a un bloqueo aurículoventricular avanzado o la aparición de sintomatología.

En el presente reporte se ha descrito y analizado la bradicardia sinusal como efecto adverso de la quimioterapia, más frecuente en la leucemia mielocítica aguda; la droga relacionada a la bradicardia más común fue la citarabina combinada con daunorubicina.

\section{Responsabilidades éticas}

Protección de personas y animales. Los autores declaran que para esta investigación no se han realizado experimentos en seres humanos ni en animales.

Confidencialidad de los datos. Los autores declaran que han seguido los protocolos de su centro de trabajo sobre la publicación de datos de pacientes.

Derecho a la privacidad y consentimiento informado. Los autores declaran que en este artículo no aparecen datos de pacientes.

\section{Contribuciones de los autores}

Ruiz-Moria E, Ayala-Bustamantea L, Burgos-Bustamantea J y Román CP participaron en la concepción, realización, redacción y revisión de la versión final.

\section{Financiación}

Ninguna.

\section{Conflictos de interés}

Ninguno.

\section{Bibliografía}

1. Allemani C, Weir HK, Carreira H. Global surveillance of cancer survival 1995-2009: analysis of individual data for 25,676,887 patients from 279 population-based registries in 67 countries (CONCORD-2). Lancet. 2015;385:977-1010.

2. Brana I, Tabernero J. Cardiotoxicity. Ann Oncol. 2010;21, vii1739.

3. Tamargo J, Caballero R, Delpon E. Cancer chemotherapy and cardiac arrhythmias: a review. Drug Saf. 2015;38:129-52.

4. Markman TM, Nazarian S. Arrhythmia and electrophysiological effects of chemotherapy: a review. Oncology. 2016;91:61-8.

5. Guglin M, Aljayeh M, Saiyad S, Ali R, Curtis AB. Introducing a new entity: chemotherapy-induced arrhythmia. Europace. 2009;11:1579-86.

6. Farmakis D, Parissis J, Filippatos G. Insights into oncocardiology: atrial fibrillation in cancer. J Am Coll Cardiol. 2014;63:945-53.

7. O'Neal WT, Lakoski SG, Qureshi W, Judd SE, Howard G, Howard VJ, et al. Relation between cancer and atrial fibrillation (from the REasons for Geographic And Racial Differences in Stroke Study). Am J Cardiol. 2015;115:1090-4. 
8. Dubner S, Moss A, Pérez A, Schapachnik E. El síndrome del intervalo QT prolongado desde el punto de vista de un cardiólogo. Rev Arg Cardiol. 2004;72:467-73.

9. Ruiz-Mori E, Ayala L, Burgos J. Insuficiencia cardiaca por quimioterapia. Rev Soc Peruana Med Interna. 2016;29:59-64.

10. Parma G, Lluberas N, Castillo C, Ormaechea G. Quimioterápicos y cardiotoxicidad: un enfoque actual y práctico para el clínico de una disciplina en pleno desarrollo. Arch Med Int. 2013;35:37-47.

11. Kalil Filho R, Haijar LA, Bacal F, Hoff PM, Diz M, del P, Galas FRBG, et al. I Diretriz Brasileira de Cardio-Oncologia da Sociedade Brasileira de Cardiologia. Arq Bras Cardiol. 2011;96 2 supl.1:1-52.

12. Cil T, Kaplan MA, Altintas A, Pasa S, Isikdogan A. Cytosinearabinoside induced bradycardia in patient with non-Hodgkin lymphoma: a case report. Leuk Lymphoma. 2007;48:1247-9.

13. Hirvonen H, Salmi T, Heinonen E, Antila K, Valimaki I. Vincristine treatment of acute lymphoblastic leukemia induces transient autonomic cardioneuropathy. Cancer. 1989;64:801-5.

14. Nazir H, AlFutaisi A, Zacharia M, Elshinawy M, Mevada S, Alrawas A, et al. Vincristine-induced neuropathy in pediatric patients with acute lymphoblastic leukemia in Oman: Frequent autonomic and more severe cranial nerve involvement. Pediatr Blood Cancer. 2017;64:e26677, https://doi.org/10.1002/pbc.26677.

15. Ever MS, Von Hoff DD, Benjamin RS. A historical perspective of anthracycline cardiotoxicity. Heart Fail Clin. 2011;7:363-72.

16. Kilickap S, Akgul E, Aksoy S, Aytemir K, Barista I. Doxorubicininduced second degree and complete atrioventricular block. Europace. 2005;7:227-30.
17. Xie Z, Wu W, Xing H, Cai Y, Wang X, Chen J. Bradycardia associated with pegylated liposomal doxorubicin administration: a case report. Eur J Hosp Pharm. 2017;24: 128-30.

18. Jimenez P, Pathak A, Phan A. The role of taxanes in the management of gastroesphageal cancer. J Gastrointest Oncol. 2011;2:240-9.

19. Kenyon BM, et al. Effects of thalidomide and related metabolites in a mouse corneal model of neovascularization. Exp Eye Res. 1997;64:971-8.

20. Fahdi IE, Gaddam V, Saucedo J, Kishan C, Vyas K, Deneke M, et al. Bradycardia during therapy for multiple myeloma with thalidomide. Am J Cardiol. 2004;93:1052-5.

21. López-de la Cruz I, Aguayo-González A, López-Karpovitch X. Bradicardia asociada al uso de talidomida en pacientes con enfermedades hematológicas: experiencia en una sola institución. Rev Invest Clin. 2006;58: 424-31.

22. Rajkumar SV, Gertz MA, Lacy MQ, Dispenzieri A, Fonseca R, Geyer SM, et al. Thalidomide as initial therapy for early-stage mieloma. Leukemia. 2003;17:775-9.

23. Schlumbrecht M, Hehr K. Cisplatin-induced bradycardia and the importance of the QT interval. J Oncol Pharm Pract. 2015;21:157-60.

24. Yavas O, Aytemir K, Celik I. The prevalence of silent arrhythmia in patients receiving cisplatin-based chemotherapy. Turk $\mathrm{J}$ Cancer. 2008;38:12-5.

25. Suter T, Ewer M. Cancer drugs and the heart: importance and management. Eur Heart J. 2013;34:1102-11. 